\title{
Machine learning method for the management of acute kidney injury: more than just treating biomarkers individually
}

\author{
Zhongheng Zhang*,1 (iD) \\ ${ }^{1}$ Department of Emergency Medicine, Sir Run Run Shaw Hospital, Zhejiang University School of Medicine, Hangzhou, 310016, PR \\ China \\ *Author for correspondence: zh_zhang1984@zju.edu.cn
}
“6 the ML approach provides a large number of mathematical algorithms to help to establish and realize precise medicine in the management of AKI."

First draft submitted: 18 August 2019; Accepted for publication: 23 August 2019; Published online: 27 September 2019

Keywords: acute kidney injury $\bullet$ big data $\bullet$ biomarkers $\bullet$ critical care $\bullet$ machine learning $\bullet$ prediction

Acute kidney injury (AKI) is frequently encountered in the intensive care unit (ICU). The occurrence of AKI is of clinical importance because there is strong evidence that even a mild increase in serum creatinine is associated with significantly increased risk of mortality [1]. The clinical definition of AKI is largely dependent on serum creatinine and urine output. However, the increase in serum creatinine usually indicates that the renal function has been severely impaired, and interventions to protect renal functions are usually delayed based on this definition. Moreover, the serum levels of creatinine can be influenced by multiple factors other than renal function. Thus, strenuous efforts have been made to predict AKI accurately and timely in high-risk patients. Many novel biomarkers were proposed to substitute creatinine in the evaluation of renal function, such as cystatin C [2], NGAL, TIM-2, KIM-1 and IGFBP-7 [3]. Most of these biomarkers were investigated independently, and their diagnostic performances were found to be moderate to high (area under the curve: 0.87; 95\% CI: 0.81-0.93). However, an individual biomarker is limited in its prediction for AKI, in other words, the development of AKI is multifactorial. I propose that the predictive performance can be further improved with the use of advanced machine learning (ML) techniques.

Recent years have witnessed rapid advances in the ML techniques. Generally speaking, the ML approach comprises three broad categories according to their purposes. Supervised learning refers to the algorithm to train a function mapping from feature space to the label (outcome). All observations (subjects) should be labeled in advance. Some sophisticated supervised ML methods include neural networks, decision trees, random forest and regression models. The task to develop a model for the prediction of AKI also belongs to the supervised ML. The advantage of ML such as neural network and decision trees is that they can automatically handle the nonlinear terms, as well as interactions among features. In the era of big data, tens of thousands of features of a subject can be collected and the complex relationships cannot be fully captured with the conventional regression model. The disadvantage of these ML methods are that the underlying relationship between covariates and outcome cannot be fully understand by human beings [4]. Conventionally, generalized linear model is employed to develop risk prediction models in clinical medicine. The advantage of such kind of models is that the coefficient of each covariate can be intuitively interpreted because the exponentiation of the coefficient gives the odds ratio $\left(e^{\beta}=\right.$ odds ratio). In this vein, the models also help to understand the causal relationship between covariates and outcome. The ML methods are widely used for the prediction of AKI in critical care setting. Supplementary Table 1 lists studies employing ML to predict AKI published in 2019 [5-11]. In these studies, various ML methods such as gradient boosted tree, k-nearest neighbors and Naive Bayes were used to predict AKI. The reported accuracies and area under the curves were quite different among the studies, which was attributable to the differences in the study population, the predictors, validation methods and ML algorithms. A systematic review and meta-analysis can be helpful in exploring the potential heterogeneity of the high-risk patients. 
The second method is the unsupervised ML, which are typically trained in unlabeled observations. Intuitively, unsupervised ML aims to identify latent subclasses by features of the subjects, and there is no supervisor to guide the training iteration. Unsupervised ML encompasses principal component analysis, clustering analysis, latent profile (class) analysis and latent Markov models [12-14]. The unsupervised ML plays an important role in the exploration of the heterogeneity in clinical researches. Although the randomized controlled trial only reported an average effect in the study population, the latent class analysis can help to discover underlying latent subgroups that respond differently to specific interventions. For example, Bhatraju et al. identified two molecularly distinct AKI subphenotypes with different clinical outcomes and responses to vasopressin therapy [15]. Such subphenotypes have important clinical relevance because different subphenotypes respond differently to interventions. Moreover, clinical trials testing different drugs can be performed in different subphenotypes.

The third category of ML is the reinforcement learning (RL) method, in which the agent receives a reward after taking an action in a certain state. Thus, the value of a reward is a function of the action and state space. The aim of the agent is to maximize the long-term reward, also known as the return, by responding properly to the state space. RL has been successfully used to individualize treatment strategy for the management of sepsis and mechanical ventilation $[16,17]$. However, to the best of my knowledge, RL has never been used in the management of AKI. $\mathrm{RL}$ is a robust algorithm that can help to form a heuristic approach in the management of AKI. In the design of an AKI study with RL method, the state space can be constructed by using demographics, laboratory variables, hemodynamics and vital signs, and the action space can be formed by vasopressors, fluid strategy and diuretics [18]. In this framework, the heuristic treatment strategy is explored as a whole, which is different from the conventional approach that each specific intervention is explored individually.

Due to the rapid development of the ML technique in computer science, it will certainly advance the clinical practice to facilitate patient management. Many modern ICUs have equipped with the early warning system that can help clinicians to identify high-risk patients. However, many of these early warning systems are based on knowledge and fixed rules, but not based on artificial intelligence. In the future, the prediction model established by sophisticated $\mathrm{ML}$ algorithms can be more readily to adapt to a certain working environment (i.e., it can learn from the big data provided from electronic healthcare records) and more accurately predict clinical outcomes. Furthermore, these algorithms can also help to recommend individualized treatment strategy if the RL algorithm is deployed in the ICU.

In conclusion, the ML approach provides a large number of mathematical algorithms to help to establish and realize precise medicine in the management of AKI. Clinical investigators should familiarize themselves to the rapidly developing field so that best ML model can be tailored to a specific clinical scenario.

\section{Supplementary data}

To view the supplementary data that accompany this paper please visit the journal website at: www.futuremedicine.com/doi/sup $\mathrm{pl} / 10.2217 / \mathrm{bmm}-2019-0363$

\section{Financial \& competing interests disclosure}

Z Zhang received funding from Zhejiang Engineering Research Center of Intelligent Medicine (grant number 2016E10011) from the First Affiliated Hospital of Wenzhou Medical University, The public welfare research project of Zhejiang province (grant number LGF18H150005), the National Natural Science Foundation of China (grant number 81901929) and Scientific research project of Zhejiang Education Commission (grant number Y201737841). The author has no other relevant affiliations or financial involvement with any organization or entity with a financial interest in or financial conflict with the subject matter or materials discussed in the manuscript apart from those disclosed.

No writing assistance was utilized in the production of this manuscript.

\section{References}

1. Kidney Disease Improving Global Outcomes Acute Kidney Injury Work Group. KDIGO clinical practice guideline for acute kidney injury. Kidney Int. 2(Suppl. 1), 1-138 (2012).

2. Zhang Z, Lu B, Sheng X, Jin N. Cystatin C in prediction of acute kidney injury: a systemic review and meta-analysis. Am. J. Kidney Dis. 58(3), 356-365 (2011).

3. Beker BM, Corleto MG, Fieiras C, Musso CG. Novel acute kidney injury biomarkers: their characteristics, utility and concerns. Int. Urol. Nephrol. 50(4), 705-713 (2018). 
4. Zhang Z, Beck MW, Winkler DA et al. Opening the black box of neural networks: methods for interpreting neural network models in clinical applications. Ann. Transl. Med. 6(11), 216-216 (2018).

5. Parreco J, Soe-Lin H, Parks JJ et al. Comparing machine learning algorithms for predicting acute kidney injury. Am. Surg. 85(7), 725-729 (2019).

6. Tran NK, Sen S, Palmieri TL et al. Artificial intelligence and machine learning for predicting acute kidney injury in severely burned patients: a proof of concept. Burns 45(6), 1350- 1358 ( 2019).

7. He J, Hu Y, Zhang X, Wu L, Waitman LR, Liu M. Multi-perspective predictive modeling for acute kidney injury in general hospital populations using electronic medical records. JAMIA Open 2(1), 115-122 (2019).

8. Adhikari L, Ozrazgat-Baslanti T, Ruppert $\mathrm{M}$ et al. Improved predictive models for acute kidney injury with IDEA: Intraoperative Data Embedded Analytics. PLoS ONE 14(4), e0214904 (2019).

9. Zimmerman LP, Reyfman PA, Smith ADR et al. Early prediction of acute kidney injury following ICU admission using a multivariate panel of physiological measurements. BMC Med. Inform. Decis. Mak. 19(Suppl. 1), 16-12 (2019).

10. Ibrahim NE, McCarthy CP, Shrestha $S$ et al. A clinical, proteomics, and artificial intelligence-driven model to predict acute kidney injury in patients undergoing coronary angiography. Clin. Cardiol. 42(2), 292-298 (2019).

11. Tomašev N, Glorot X, Rae JW et al. A clinically applicable approach to continuous prediction of future acute kidney injury. Nature 572(7767), 116-119 (2019).

12. Collins LM, Lanza ST. Latent class and latent transition analysis: With applications in the social, behavioral, and health sciences. John Wiley \& Sons, Inc., NJ, USA (2009).

13. Zhang Z, Abarda A, Contractor AA, Wang J, Dayton CM. Exploring heterogeneity in clinical trials with latent class analysis. Ann. Transl. Med. 6(7), 119-119 (2018).

14. Pennoni F, Barbato M, Del Zoppo S. A latent Markov model with covariates to study unobserved heterogeneity among fertility patterns of couples employing natural family planning methods. Front. Public Health 5, 186 (2017).

15. Bhatraju PK, Zelnick LR, Herting J et al. Identification of acute kidney injury subphenotypes with differing molecular signatures and responses to vasopressin therapy. Am. J. Respir. Crit. Care Med. 199(7), 863-872 (2019).

16. Komorowski M, Celi LA, Badawi O, Gordon AC, Faisal AA. The Artificial Intelligence Clinician learns optimal treatment strategies for sepsis in intensive care. Nat. Med. 24(11), 1716-1720 (2018).

17. Prasad N, Cheng L-F, Chivers C, Draugelis M, Engelhardt BE. A reinforcement learning approach to weaning of mechanical ventilation in intensive care units. ArXiv. 19(Suppl. 2), 57 (2017)

18. Silver D, Huang A, Maddison CJ et al. Mastering the game of Go with deep neural networks and tree search. Nature 529(7587), 484-489 (2016). 
